\title{
Probiotics and Time to Achieve Full Enteral Feeding in Human Milk-Fed and Formula-Fed Preterm Infants: Systematic Review and Meta-Analysis
}

\author{
Arianna Aceti 1,2, Davide Gori ${ }^{2,3}$, Giovanni Barone ${ }^{2,4}$, Maria Luisa Callegari 2,5 , \\ Maria Pia Fantini 2,3, Flavia Indrio 2,6,7, Luca Maggio 2,4,7, Fabio Meneghin 2,8, \\ Lorenzo Morelli 2,5, Gianvincenzo Zuccotti ${ }^{2,9}$ and Luigi Corvaglia ${ }^{1,2,7, *}$ \\ 1 Neonatology and Neonatal Intensive Care Unit, Department of Medical and Surgical Sciences (DIMEC), \\ University of Bologna, S.Orsola-Malpighi Hospital, Bologna 40138, Italy; arianna.aceti2@unibo.it \\ 2 Task Force on Probiotics of the Italian Society of Neonatology, Milan 20126, Italy; \\ dedegori27@gmail.com (D.G.); gbarone85@yahoo.it (G.B.); marialuisa.callegari@unicatt.it (M.L.C.); \\ mariapia.fantini@unibo.it (M.P.F.); f.indrio@alice.it (F.I.); luca.maggio@fastwebnet.it (L.Ma.); \\ fabio.meneghin@asst-fbf-sacco.it (F.M.); lorenzo.morelli@unicatt.it (L.Mo.); \\ gianvincenzo.zuccotti@unimi.it (G.Z.) \\ 3 Department of Biomedical and Neuromotor Sciences (DIBINEM), University of Bologna, \\ Bologna 40138, Italy \\ 4 Neonatal Unit, Catholic University, Rome 00168, Italy \\ 5 Institute of Microbiology, UCSC, Piacenza 29122, Italy \\ 6 Department of Pediatrics, Aldo Moro University, Bari 70124, Italy \\ 7 Study Group of Neonatal Gastroenterology and Nutrition of the Italian Society of Neonatology, \\ Milan 20126, Italy \\ 8 Division of Neonatology, Children Hospital V. Buzzi, ICP, Milan 20154, Italy \\ 9 Department of Pediatrics, Children Hospital V. Buzzi, University of Milan, Milan 20154, Italy \\ * Correspondence: luigi.corvaglia@unibo.it; Tel./Fax: +39-051-342-754
}

Received: 13 June 2016; Accepted: 26 July 2016; Published: 30 July 2016

\begin{abstract}
Probiotics have been linked to a reduction in the incidence of necrotizing enterocolitis and late-onset sepsis in preterm infants. Recently, probiotics have also proved to reduce time to achieve full enteral feeding (FEF). However, the relationship between FEF achievement and type of feeding in infants treated with probiotics has not been explored yet. The aim of this systematic review and meta-analysis was to evaluate the effect of probiotics in reducing time to achieve FEF in preterm infants, according to type of feeding (exclusive human milk (HM) vs. formula). Randomized-controlled trials involving preterm infants receiving probiotics, and reporting on time to reach FEF were included in the systematic review. Trials reporting on outcome according to type of feeding (exclusive HM vs. formula) were included in the meta-analysis. Fixed-effect or random-effects models were used as appropriate. Results were expressed as mean difference (MD) with $95 \%$ confidence interval (CI). Twenty-five studies were included in the systematic review. In the five studies recruiting exclusively HM-fed preterm infants, those treated with probiotics reached FEF approximately 3 days before controls (MD -3.15 days (95\% CI $-5.25 /-1.05), p=0.003$ ). None of the two studies reporting on exclusively formula-fed infants showed any difference between infants receiving probiotics and controls in terms of FEF achievement. The limited number of included studies did not allow testing for other subgroup differences between HM and formula-fed infants. However, if confirmed in further studies, the 3-days reduction in time to achieve FEF in exclusively HM-fed preterm infants might have significant implications for their clinical management.
\end{abstract}

Keywords: probiotics; preterm infants; human milk; full enteral feeding; systematic review 


\section{Introduction}

Nutrition during critical time windows in early life can affect long-term health [1]. Early provision of optimal enteral nutrition to preterm infants might improve neurodevelopmental outcome by decreasing the rate of several complications of prematurity, such as extrauterine growth restriction, necrotizing enterocolitis (NEC), sepsis, bronchopulmonary dysplasia, and retinopathy of prematurity [2].

Late introduction and slow advancement of enteral feeding may alter gastrointestinal motility and disrupt microbial colonization [3], leading to a delay in establishing full enteral feeding (FEF). The consequent prolonged need for parenteral nutrition can have serious infectious and metabolic complications, which might prolong hospital stay, increase morbidity and mortality, and affect growth and development [4].

Several clinical variables and interventions have been proposed as predictors of the time to FEF achievement in preterm and very-low-birth-weight (VLBW) infants. Among these variables, the influence of type of feeding was also documented, as FEF achievement was delayed in formula-fed infants compared to human milk (HM)-fed infants [5].

Recently, probiotic use has been associated with a reduced time to achieve FEF and better feeding tolerance [6], as well as a reduction of NEC [7,8] and late-onset sepsis [9]. Probiotics are live microorganisms which, when ingested in adequate amounts, confer a health benefit to the host, by modifying the composition and function of gut microbiota and the immunological responses in the host [10]. The role of probiotics in attaining a more rapid achievement of FEF could be related to their favorable effect on the physiological intestinal dysbiosis of preterm infants [11], which is the result of the exposure to a unique environment and to several iatrogenic manipulations, such as broad spectrum antibiotics [12]. It is well known that gut microbiota in HM-fed infants is different compared to formula-fed infants [13]; data from an observational study also suggest a feeding-dependent effect of probiotics, as in that study NEC incidence was reduced in infants treated with probiotics and receiving HM, but not in those exclusively formula-fed [14]. However, the relationship between probiotics and type of feeding in attaining a more rapid achievement of FEF has not been explored yet, even in the most recent meta-analysis on this topic [6].

Thus, the aim of the present paper was to evaluate the effect of probiotics on time to FEF achievement according to type of feeding (exclusive HM vs. formula), by performing a systematic review and meta-analysis of currently available literature on this topic.

\section{Materials and Methods}

\subsection{Literature Search}

The study protocol was designed by the members of the Task Force on Probiotics of the Italian Society of Neonatology. PRISMA guidelines [15] were followed in order to perform a systematic review of published studies reporting the relationship between probiotic use and time to FEF achievement in preterm infants according to type of feeding.

In order to be included in the meta-analysis, studies had to meet the following inclusion criteria: randomized or quasi-randomized clinical trials involving preterm infants (gestational age (GA) <37 weeks) who received, within one month of age, any probiotic compared to placebo or no treatment, and reporting on type of feeding. The outcome of interest was time for FEF achievement (any definition). Only English-written studies and studies involving humans were included in the meta-analysis.

A search was conducted for studies published before 2 March 2016 in PubMed [16], the Cochrane Library [17], and Embase [18]. The following search string was used for the PubMed search: ((preterm infant OR pre-term infant) OR (preterm infants OR pre-term infants) OR (preterm neonate OR pre-term neonate) OR (preterm neonates OR pre-term neonates) OR (preterm newborn OR pre-term newborn) OR (preterm newborns OR pre-term newborns) OR (premature infant OR premature infants) OR 
(premature neonate OR premature neonates) OR (premature newborn OR premature newborns) OR infant, extremely premature (MeSH Heading (MH)) OR premature birth (MH) OR infant, low birth weight (MH) OR infant, very low birth weight (MH)) AND (full enteral* OR feed*) AND (probiotic OR probiotics OR pro-biotic OR pro-biotics OR probio*)) NOT (animals (MH) NOT humans (MH).

The string was built up by combining all the terms related to probiotics and FEF achievement: PubMed MeSH terms, free-text words, and their combinations obtained through the most proper Boolean operators were used. The same criteria were used for searching the Cochrane Library and Embase.

Arianna Aceti and Luigi Corvaglia performed the literature search: relevant studies were identified from the abstract; full-texts of relevant studies were examined, as well as their reference lists in order to identify additional studies.

\subsection{Data Extraction and Meta-Analysis}

Study details (population, characteristics of probiotic and placebo, type of feeding, and outcome assessment) were evaluated independently by Arianna Aceti and Luigi Corvaglia, and checked by Davide Gori. Study quality was evaluated independently by Arianna Aceti and Davide Gori using the risk of bias tool as proposed by the Cochrane collaboration (Chapter 8 of the Cochrane Handbook of Systematic Reviews) [19].

The corresponding authors of the studies in which days to FEF achievement were not reported as mean \pm standard deviation (SD) were contacted by email. When data were not provided, the study was not included in the meta-analysis.

The association between probiotic use and FEF achievement according to type of feeding was evaluated by a meta-analysis conducted by AA and DG using the RevMan software (Cochrane Informatics and Knowledge Management Department, version 5.3.5) downloaded from the Cochrane website [20]. Mean difference (MD) in days to achieve FEF between infants receiving probiotics and those receiving placebo or no treatment was calculated using the inverse variance method, and reported with $95 \%$ confidence interval $(\mathrm{CI})$.

For the analysis, we planned to use at first a fixed effect model. Heterogeneity was measured using the $I^{2}$ test: if significant heterogeneity was present ( $p<0.05$ from the $\chi^{2}$ test) and/or the number of studies was $\leqslant 5$, a random-effects model was used instead.

\section{Results}

\section{Literature Search}

Overall, 372 papers were identified through the literature search, 155 in PubMed [16], 73 in the Cochrane Library [17], and 144 in Embase [18].

As shown in Figure 1, 35 studies met the inclusion criteria [21-55]. Fourteen additional papers were identified from the reference lists of included studies or by "snowballing" techniques [52,56-68].

Twenty-four studies were excluded after examining the full-texts $[28,29,31-33,35,42-47,51,53-$ $55,57-59,62,63,65,69]$. Twenty-five studies were then suitable for inclusion in the systematic review (Table 1) [21-27,30,34,36-41,48-50,56,60,61,64,66,68,70].

Among them, only eight studies reported FEF achievement according to type of feeding: infants were fed exclusively HM, either own mother's (OMM) or donor human milk (DHM), in six studies [22,38,50,56,60,70], while two studies reported FEF in exclusively formula-fed infants [41,61].

The corresponding authors of four of these papers were contacted by email, as data for FEF achievement were not suitable for inclusion in the meta-analysis: mean \pm SD of days for FEF achievement were provided for one study [22], while data were unavailable for three studies [41,61,70]; these three studies were thus excluded from the meta-analysis.

Overall, five studies were included in the meta-analysis: in all these studies, infants were fed exclusively HM, either OMM or DHM (Figure 1) [22,38,50,56,60]. 


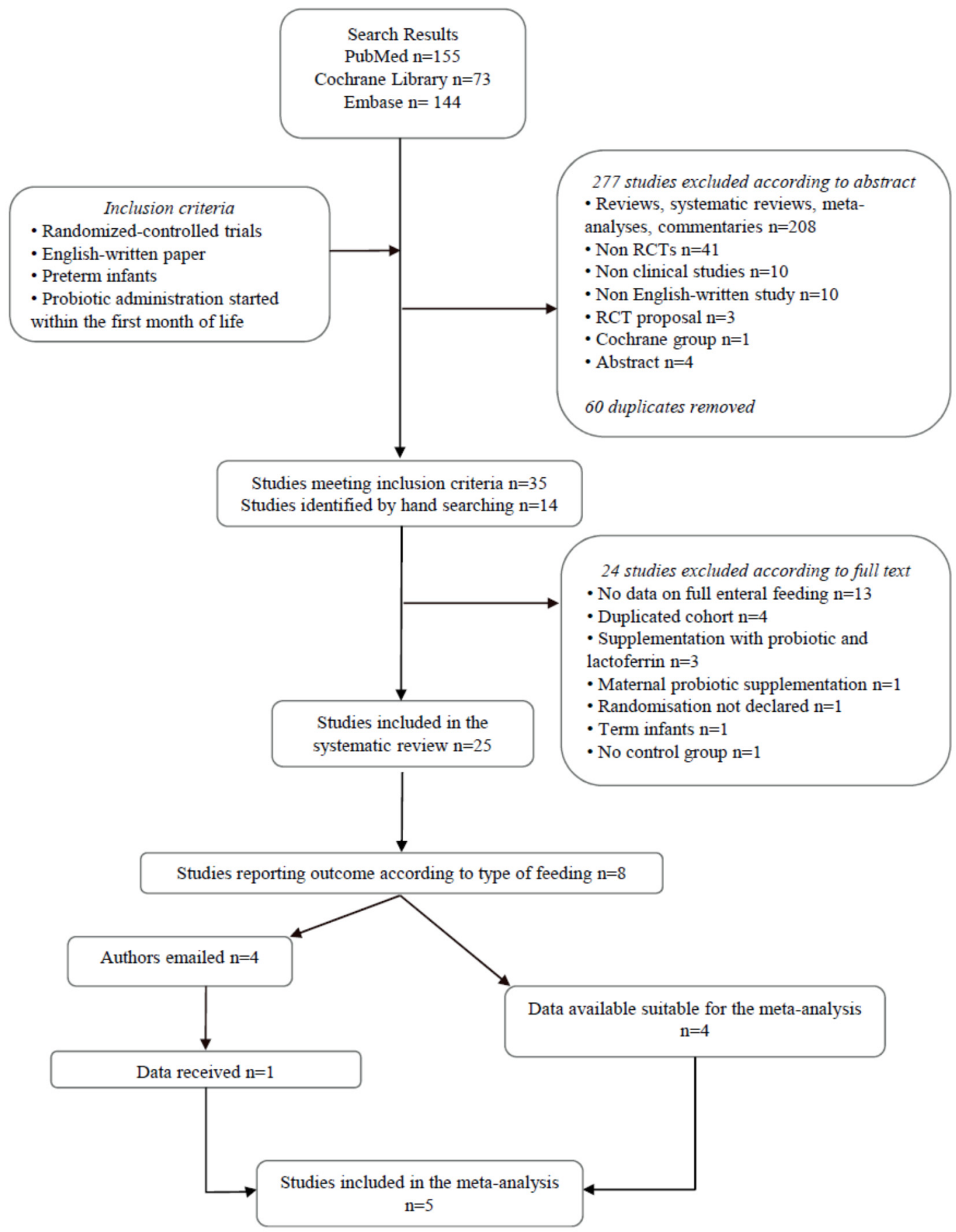

Figure 1. Flow chart of the search strategy used for the systematic review. The relevant number of papers at each point is given. 
Table 1. Studies included in the systematic review.

\begin{tabular}{|c|c|c|c|c|c|c|}
\hline \multirow{4}{*}{ Author, Year } & \multirow{4}{*}{ Study Details } & \multirow{4}{*}{ Study Population } & Intervention Specie & \multirow{4}{*}{ Milk } & \multirow{4}{*}{ Placebo } & \multirow{4}{*}{ FEF Definition } \\
\hline & & & Dose (D) & & & \\
\hline & & & Start of Treatment (S) & & & \\
\hline & & & End of Treatment (E) & & & \\
\hline \multirow{4}{*}{ Bin-Nun, 2005 [40] } & $\mathrm{P}$ & \multirow{4}{*}{$\begin{array}{l}\text { Preterm infants with } \mathrm{BW}<1500 \mathrm{~g} \text {, who } \\
\text { began enteral feeding on a weekday }\end{array}$} & B. infantis, Str. thermophilus, B. bifidus & \multirow{4}{*}{ OMM, PFM } & \multirow{4}{*}{$\mathrm{HM}$ or FM } & \multirow{4}{*}{$100 \mathrm{~mL} / \mathrm{kg} /$ day } \\
\hline & B & & D: $0.35 \times 10^{9}$ CFU each, OD & & & \\
\hline & $\mathrm{R}$ & & S: start of enteral feeding & & & \\
\hline & C & & E: $36 \mathrm{w}$ postconceptual age & & & \\
\hline \multirow{4}{*}{ Braga, $2011[60]$} & $\mathrm{P}$ & \multirow{4}{*}{ Inborn infants with BW 750-1499 g } & L. casei, B. Breve & \multirow{4}{*}{ HM ( \pm PFM from w3) } & \multirow{4}{*}{ Extra HM } & \multirow{4}{*}{$150 \mathrm{~mL} / \mathrm{kg} /$ day } \\
\hline & DB & & D: $3.5 \times 10^{7} \mathrm{CFU}$ to $3.5 \times 10^{9} \mathrm{CFU}$ OD & & & \\
\hline & $\mathrm{R}$ & & S: day 2 & & & \\
\hline & C & & $\begin{array}{l}\text { E: day 30, NEC diagnosis, discharge, death, } \\
\text { whichever occurred first }\end{array}$ & & & \\
\hline \multirow{4}{*}{ Costalos, 2003 [41] } & $\mathrm{P}$ & GA $28-32 \mathrm{w}$ & Saccharomyces boulardii & \multirow{4}{*}{ PFM } & \multirow{4}{*}{ MDX } & \multirow{4}{*}{ Not defined } \\
\hline & $\mathrm{R}$ & No major GI problem & D: $1 \times 10^{9}$ CFU BD & & & \\
\hline & $\mathrm{C}$ & Not receiving antibiotics & S: non-specified & & & \\
\hline & & Not receiving breast milk & $\begin{array}{l}\text { Median duration of probiotic supplementation: } \\
30 \text { days }\end{array}$ & & & \\
\hline \multirow{5}{*}{ Costeloe, 2015 [64] } & $\mathrm{P}$ & \multirow{5}{*}{$\begin{array}{c}\text { Preterm infants with } \\
\text { GA 23-30 + } 6 \text { weeks, without any lethal } \\
\text { malformation or any malformation of } \\
\text { the GI tract }\end{array}$} & Bifidobacterium breve BBG-001 & \multirow{5}{*}{ OMM, DHM, FM } & \multirow{5}{*}{ Corn starch powder } & \multirow{5}{*}{$150 \mathrm{~mL} / \mathrm{kg} /$ day } \\
\hline & DB & & D: $8 \cdot 3-8 \cdot 8 \log _{10}$ CFU/day & & & \\
\hline & $\mathrm{R}$ & & S: as soon as possible after randomisation & & & \\
\hline & C & & E: $36 \mathrm{w}$ PMA or discharge if sooner & & & \\
\hline & Multic. & & & & & \\
\hline \multirow{4}{*}{ Demirel, 2013 [27] } & $\mathrm{P}$ & \multirow{4}{*}{$\begin{array}{l}\text { Preterm infants with } \mathrm{GA} \leqslant 32 \text { weeks } \\
\text { and } \mathrm{BW} \leqslant 1500 \mathrm{~g} \text {, who survived to } \\
\text { feed enterally }\end{array}$} & S. boulardii & \multirow{4}{*}{$\mathrm{HM}, \mathrm{FM}$} & \multirow{4}{*}{ None } & \multirow{4}{*}{ Not defined } \\
\hline & $\mathrm{B}$ & & D: $5 \times 10^{9} \mathrm{CFU}$ OD & & & \\
\hline & $\mathrm{R}$ & & S: first feed & & & \\
\hline & $\mathrm{C}$ & & E: discharge & & & \\
\hline \multirow{5}{*}{ Dilli, 2015 [49] } & $P$ & & B. lactis & & & $\begin{array}{c}100 \mathrm{~mL} / \mathrm{kg} / \text { day } \\
\text { (FEF for hydration) }\end{array}$ \\
\hline & DB & $\begin{array}{l}\text { Preterm infants with } G A<32 \text { weeks and } \\
\text { BW }<1500 \mathrm{~g} \text {, born at or transferred to } \\
\text { the NICU within the first week of life }\end{array}$ & D: $5 \times 10^{9} \mathrm{CFU}$ & HM, FM & MDX powder & $\begin{array}{l}150 \mathrm{~mL} / \mathrm{kg} / \text { day } \\
\text { (FEF for growth) }\end{array}$ \\
\hline & $\mathrm{R}$ & and fed enterally before inclusion & S: beyond d7 after birth & & & \\
\hline & $\mathrm{C}$ & & E: death or discharge (max 8 weeks) & & & \\
\hline & Multic. & & & & & \\
\hline
\end{tabular}


Table 1. Cont

\begin{tabular}{|c|c|c|c|c|c|c|}
\hline \multirow{4}{*}{ Author, Year } & \multirow{4}{*}{ Study Details } & \multirow{4}{*}{ Study Population } & Intervention Specie & \multirow{4}{*}{ Milk } & \multirow{4}{*}{ Placebo } & \multirow{4}{*}{ FEF Definition } \\
\hline & & & Dose (D) & & & \\
\hline & & & Start of Treatment (S) & & & \\
\hline & & & End of Treatment (E) & & & \\
\hline \multirow{4}{*}{$\begin{array}{l}\text { Fernández-Carrocera, } \\
2013[30]\end{array}$} & $P$ & Preterm infants with & $\begin{array}{c}\text { L. acidophilus } 1 \times 10^{9} \mathrm{CFU} / \mathrm{g} \text {, L. rhamnosus } \\
4.4 \times 10^{8} \mathrm{CFU} / \mathrm{g}, \mathrm{L} \text {. casei } 1 \times 10^{9} \mathrm{CFU} / \mathrm{g} \text {, } \\
\text { L. plantarum } 1.76 \times 10^{8} \mathrm{CFU} / \mathrm{g} \text {, B. infantis } \\
2.76 \times 10^{7} \mathrm{CFU} / \mathrm{g} \text {, Str. thermophilus } \\
\quad 6.6 \times 10^{5} \mathrm{CFU} / \mathrm{g}\end{array}$ & \multirow{4}{*}{ OMM, PFM } & \multirow{4}{*}{ None } & \multirow{4}{*}{ Not defined } \\
\hline & DB & $\mathrm{BW}<1500 \mathrm{~g}$ & Total D: $1 \mathrm{~g}$ powder OD & & & \\
\hline & $\mathrm{R}$ & $\begin{array}{l}\text { Infants with NEC stage IA and stage IB } \\
\text { were excluded }\end{array}$ & S: start of enteral feeding & & & \\
\hline & $\mathrm{C}$ & & E: non specified & & & \\
\hline \multirow{6}{*}{ Hays, 2014 [66] } & $\mathrm{P}$ & $\begin{array}{c}\text { Preterm infants with GA } 25-31 \text { weeks, } \\
\text { BW 700-1600 g, AGA, enteral feeding } \\
\text { initiated before day } 5\end{array}$ & Probiotic group composed of 3 subgroups: & \multirow{6}{*}{ OMM, DM or PFM } & \multirow{6}{*}{ MDX } & \multirow{6}{*}{ Not defined } \\
\hline & DB & \multirow{5}{*}{$\begin{array}{l}\text { Infants with NEC stage } \geqslant I B \\
\text { malformations or severe medical or } \\
\text { surgical conditions were excluded }\end{array}$} & P1 B. lactis & & & \\
\hline & $\mathrm{R}$ & & P2 B. longum & & & \\
\hline & C & & P3 B. lactis + longum & & & \\
\hline & \multirow[b]{2}{*}{ Multic. } & & D: $1 \times 10^{9} \mathrm{CFU}$ each probiotic daily & & & \\
\hline & & & $\begin{array}{c}\text { Duration: } 4 \text { weeks for infants } \geqslant 29 \mathrm{w} / 6 \text { weeks for } \\
\text { infants } \leqslant 28 \mathrm{w} \text { GA }\end{array}$ & & & \\
\hline \multirow{4}{*}{ Hikaru, 2010 [68] } & $\mathrm{P}$ & \multirow{4}{*}{$\begin{array}{l}\text { Extremely low birth weight and very } \\
\text { low birth weight infants }\end{array}$} & B. breve & \multirow{4}{*}{ OMM, PFM } & \multirow{4}{*}{ None } & \multirow{4}{*}{ Not defined } \\
\hline & $\mathrm{R}$ & & D: $0.5 \times 10^{9} \mathrm{CFU} B D$ & & & \\
\hline & C & & S: birth & & & \\
\hline & & & E: discharge from NICU & & & \\
\hline \multirow{5}{*}{ Jacobs, 2013 [25] } & $\mathrm{P}$ & \multirow{5}{*}{$\begin{array}{l}\text { Preterm infants with GA }<32 \text { weeks and } \\
\text { BW }<1500 \mathrm{~g}\end{array}$} & $\begin{array}{l}\text { B. infantis BB-02 } 300 \mathrm{CFU} \times 10^{6} \text {, Str. thermophilus } \\
\text { Th-4 } 350 \mathrm{CFU} \times 10^{6}, \text { B. lactis BB-12 } 350 \mathrm{CFU} \times 10^{6}\end{array}$ & \multirow{5}{*}{$\mathrm{HM}, \mathrm{FM}$} & \multirow{5}{*}{ MDX powder } & \multirow{5}{*}{$\begin{array}{l}\text { Enteral feeds of } \\
120 \mathrm{~mL} / \mathrm{kg} \text { for } \\
\quad \geqslant 3 \text { days }\end{array}$} \\
\hline & $\mathrm{DB}$ & & Total D: $1 \times 10^{9} \mathrm{CFU} \times 1.5 \mathrm{~g}$ MDX powder OD & & & \\
\hline & $\mathrm{R}$ & & S: enteral feed $\geqslant 1 \mathrm{~mL}$ every $4 \mathrm{~h}$ & & & \\
\hline & $\mathrm{C}$ & & E: discharge or term corrected age & & & \\
\hline & Multic. & & & & & \\
\hline
\end{tabular}


Table 1. Cont.

\begin{tabular}{|c|c|c|c|c|c|c|}
\hline \multirow{4}{*}{ Author, Year } & \multirow{4}{*}{ Study Details } & \multirow{4}{*}{ Study Population } & Intervention Specie & \multirow{4}{*}{ Milk } & \multirow{4}{*}{ Placebo } & \multirow{4}{*}{ FEF Definition } \\
\hline & & & Dose (D) & & & \\
\hline & & & Start of Treatment (S) & & & \\
\hline & & & End of Treatment (E) & & & \\
\hline \multirow{5}{*}{ Lin, 2008 [39] } & $\mathrm{P}$ & \multirow{5}{*}{$\begin{array}{c}\text { Preterm infants with } \mathrm{GA}<34 \text { weeks and } \\
\mathrm{BW} \leqslant 1500 \mathrm{~g} \text {, who survived to feed } \\
\text { enterally }\end{array}$} & $\begin{array}{c}\text { L. acidophilus NCDO 1746, B. bifidum } \\
\text { NCDO } 145310^{9} \text { CFU }\end{array}$ & \multirow{5}{*}{ HM, FM } & \multirow{5}{*}{ None } & \multirow{5}{*}{$\begin{array}{l}\text { Oral intake of } \\
100 \mathrm{~mL} / \mathrm{kg} / \mathrm{day}\end{array}$} \\
\hline & $\mathrm{B}$ & & D: $1 \times 10^{9} \mathrm{CFU}$ each probiotic $(=125 \mathrm{mg} / \mathrm{kg}) \mathrm{BD}$ & & & \\
\hline & $\mathrm{R}$ & & S: day 2 of age & & & \\
\hline & C & & Duration: 6 weeks & & & \\
\hline & Multic. & & & & & \\
\hline \multirow{4}{*}{ Manzoni, 2006 [56] } & $\mathrm{P}$ & \multirow{4}{*}{$\begin{array}{l}\text { Infants with } \mathrm{BW}<1500 \mathrm{~g}, \geqslant 3 \text { day of life, } \\
\text { who started enteral feeding with } \mathrm{HM}\end{array}$} & L. casei subspecies rhamnosus LGG & \multirow{4}{*}{ OMM, DM } & \multirow{4}{*}{ None } & \multirow{4}{*}{ Not defined } \\
\hline & DB & & D: $6 \times 10^{9} \mathrm{CFU} /$ day & & & \\
\hline & $\mathrm{R}$ & & S: day 3 of life & & & \\
\hline & C & & E: end of the 6th week or discharge & & & \\
\hline \multirow{4}{*}{ Mihatsch, 2010 [36] } & $\mathrm{P}$ & \multirow{4}{*}{$\begin{array}{l}\text { Preterm infants with } \mathrm{GA}<30 \text { weeks and } \\
\qquad \mathrm{BW} \leqslant 1500 \mathrm{~g}\end{array}$} & B. lactis BB12 & \multirow{4}{*}{ OMM, PFM } & \multirow{4}{*}{$\begin{array}{l}\text { Indistinguishable } \\
\text { powder }\end{array}$} & \multirow{4}{*}{$150 \mathrm{~mL} / \mathrm{kg} /$ day } \\
\hline & $\mathrm{R}$ & & D: $2 \times 10^{9} \mathrm{CFU} / \mathrm{kg} 6$ times a day & & & \\
\hline & C & & S: start of enteral feeding & & & \\
\hline & & & E: non specified & & & \\
\hline \multirow{4}{*}{ Oncel, 2014 [24] } & $\mathrm{P}$ & \multirow{4}{*}{$\begin{array}{l}\text { Preterm infants with } \mathrm{GA} \leqslant 32 \text { weeks } \\
\text { and } \mathrm{BW} \leqslant 1500 \mathrm{~g} \text {, who survived to } \\
\text { feed enterally }\end{array}$} & L. reuteri DSM 17938 & \multirow{4}{*}{$\mathrm{HM}, \mathrm{FM}$} & \multirow{4}{*}{ Oil base } & \multirow{4}{*}{ Not defined } \\
\hline & DB & & D: $1 \times 10^{8}$ CFU OD & & & \\
\hline & $\mathrm{R}$ & & S: first feed & & & \\
\hline & C & & E: death or discharge & & & \\
\hline \multirow{4}{*}{ Patole, 2014 [23] } & $\mathrm{P}$ & \multirow{4}{*}{$\begin{array}{l}\text { Preterm infants with } \mathrm{GA}<33 \text { weeks and } \\
\qquad \mathrm{BW}<1500 \mathrm{~g}\end{array}$} & B. breve & \multirow{4}{*}{$\mathrm{HM}, \mathrm{FM}$} & \multirow{4}{*}{ Dextrin } & \multirow{4}{*}{$\begin{array}{l}150 \mathrm{~mL} / \mathrm{kg} / \mathrm{daj} \\
\text { enteral feeding }\end{array}$} \\
\hline & DB & & $\begin{array}{c}\text { D: } 3 \times 10^{9} \mathrm{CFU} \text { OD }\left(1.5 \times 10^{9} \mathrm{CFU} \text { OD for }\right. \\
\text { newborn } \leqslant 27 \text { w until they reached } \\
50 \mathrm{~mL} / \mathrm{kg} / \text { day enteral feeds })\end{array}$ & & & \\
\hline & $\mathrm{R}$ & & S: start of enteral feed & & & \\
\hline & C & & E: corrected age of $37 w$ & & & \\
\hline & $\mathrm{P}$ & & B. longum BB536, L. rhamnosus GG BB536-LGG & & & \\
\hline & DB & $\mathrm{BW}<1500 \mathrm{~g}, \leqslant 2$ weeks of age, without & Total D: $1 \times 108 \mathrm{CFU} /$ day & & & \\
\hline Rougé, 2009 [37] & $\mathrm{R}$ & any disease other than those linked to & S: start of enteral feeding & OMM, DM or PFM & MDX & Not defined \\
\hline & $\mathrm{C}$ & 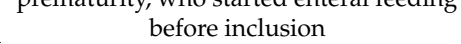 & E: discharge & & & \\
\hline & Bic. & & & & & \\
\hline
\end{tabular}


Table 1. Cont.

\begin{tabular}{|c|c|c|c|c|c|c|}
\hline \multirow{4}{*}{ Author, Year } & \multirow{4}{*}{ Study Details } & \multirow{4}{*}{ Study Population } & Intervention Specie & \multirow{4}{*}{ Milk } & \multirow{4}{*}{ Placebo } & \multirow{4}{*}{ FEF Definition } \\
\hline & & & Dose (D) & & & \\
\hline & & & Start of Treatment (S) & & & \\
\hline & & & End of Treatment (E) & & & \\
\hline \multirow{4}{*}{ Roy, 2014 [50] } & $P$ & \multirow{4}{*}{$\begin{array}{c}\text { Preterm infants (GA }<37 \text { weeks) and } \\
\text { BW }<2500 \mathrm{~g} \text {, with stable enteral feeding } \\
\text { within } 72 \mathrm{~h} \text { of birth }\end{array}$} & $\begin{array}{c}\text { L. acidophilus } 1.25 \times 10^{9} \mathrm{CFU} \times 1 \mathrm{~g}, \text { B. longum } \\
0.125 \times 10^{9} \mathrm{CFU} \times 1 \mathrm{~g}, \text { B. bifidum } \\
0.125 \times 10^{9} \mathrm{CFU} \times 1 \mathrm{~g}, \text { B. lactis } 1 \times 10^{9} \mathrm{CFU} \times 1 \mathrm{~g}\end{array}$ & \multirow{4}{*}{ HM } & \multirow{4}{*}{ Sterile water } & \multirow{4}{*}{$\begin{array}{l}120 \mathrm{~mL} / \mathrm{kg} / \text { day } \\
\text { for } \geqslant 3 \mathrm{~d}\end{array}$} \\
\hline & DB & & D: half a $1 \mathrm{~g}$ sachet & & & \\
\hline & $\mathrm{R}$ & & S: from $72 \mathrm{~h}$ of life & & & \\
\hline & C & & E: after $6 \mathrm{w}$ or at discharge & & & \\
\hline \multirow{4}{*}{$\begin{array}{l}\text { Saengtawesin, } \\
2014[48]\end{array}$} & $\mathrm{P}$ & \multirow{4}{*}{$\begin{array}{l}\text { Preterm infants with } \mathrm{GA} \leqslant 34 \text { weeks } \\
\text { and } \mathrm{BW} \leqslant 1500 \mathrm{~g}\end{array}$} & L. acidophilus $1 \times 10^{9} \mathrm{CFU}$, B. bifidum $1 \times 10^{9} \mathrm{CFU}$ & \multirow{4}{*}{ HM, PFM } & \multirow{4}{*}{ None } & \multirow{4}{*}{$150 \mathrm{~mL} / \mathrm{kg} /$ day } \\
\hline & $\mathrm{R}$ & & D: $125 \mathrm{mg} / \mathrm{kg}$ BD & & & \\
\hline & $\mathrm{C}$ & & S: start of feeding & & & \\
\hline & & & E: $6 \mathrm{w}$ of age or discharge. & & & \\
\hline \multirow{5}{*}{ Samanta, 2008 [38] } & $\mathrm{P}$ & \multirow{5}{*}{$\begin{array}{l}\text { Preterm infants with } \mathrm{GA}<32 \text { weeks and } \\
\mathrm{BW}<1500 \mathrm{~g} \text {, who started enteral } \\
\text { feeding and survived beyond } 48 \mathrm{~h} \text { of age }\end{array}$} & B. infantis, B. bifidum, B. longum, L. acidophilus & \multirow{5}{*}{$\mathrm{HM}$} & \multirow{5}{*}{ None } & \multirow{5}{*}{ Not defined } \\
\hline & DB & & & & & \\
\hline & $\mathrm{R}$ & & D: $2.5 \times 10^{9}$ CFU each probiotic, BD & & & \\
\hline & $\mathrm{C}$ & & S: start of enteral feeding & & & \\
\hline & & & E: discharge & & & \\
\hline \multirow{4}{*}{ Sari, 2011 [34] } & $\mathrm{P}$ & \multirow{4}{*}{$\begin{array}{c}\text { Preterm infants with GA }<32 \text { weeks or } \\
\text { BW }<1500 \mathrm{~g} \text {, who survived to feed } \\
\text { enterally }\end{array}$} & L. sporogenes & \multirow{4}{*}{$\mathrm{HM}, \mathrm{FM}$} & \multirow{4}{*}{ None } & \multirow{4}{*}{ Not defined } \\
\hline & B & & D: $0.35 \times 10^{9}$ CFU OD & & & \\
\hline & $\mathrm{R}$ & & S: first feed & & & \\
\hline & $\mathrm{C}$ & & E: discharge & & & \\
\hline \multirow{4}{*}{ Serce, 2013 [26] } & $\mathrm{P}$ & \multirow{4}{*}{$\begin{array}{l}\text { Preterm infants with } \mathrm{GA} \leqslant 32 \text { weeks } \\
\text { and } \mathrm{BW} \leqslant 1500 \mathrm{~g} \text {, who survived to } \\
\text { feed enterally }\end{array}$} & S. boulardii & \multirow{4}{*}{ HM, FM } & \multirow{4}{*}{ Distilled water } & $100 \mathrm{~mL} / \mathrm{kg} /$ day \\
\hline & $\mathrm{M}$ & & D: $0.5 \times 10^{9} \mathrm{CFU} / \mathrm{kg} \mathrm{BD}$ & & & enteral feeding \\
\hline & $\mathrm{R}$ & & S: non specified & & & \\
\hline & $\mathrm{C}$ & & E: non specified & & & \\
\hline \multirow{4}{*}{ Stratiki, 2007 [61] } & $\mathrm{P}$ & & Bifidobacterium lactis & & & \\
\hline & B & $\begin{array}{l}\text { Preterm infants with GA } 27-32 \text { weeks, } \\
\text { formula-fed, without major }\end{array}$ & D: $2 \times 10^{7} \mathrm{CFU} / \mathrm{g}$ of milk powder & FM & None & $150 \mathrm{~mL} / \mathrm{kg} /$ day \\
\hline & $\mathrm{R}$ & congenital anomalies & S: start of enteral feeding & & & \\
\hline & C & & E: not specified & & & \\
\hline
\end{tabular}


Table 1. Cont

\begin{tabular}{|c|c|c|c|c|c|c|}
\hline \multirow{4}{*}{ Author, Year } & \multirow{4}{*}{ Study Details } & \multirow{4}{*}{ Study Population } & Intervention Specie & \multirow{4}{*}{ Milk } & \multirow{4}{*}{ Placebo } & \multirow{4}{*}{ FEF Definition } \\
\hline & & & Dose (D) & & & \\
\hline & & & Start of Treatment (S) & & & \\
\hline & & & End of Treatment (E) & & & \\
\hline \multirow{4}{*}{ Tewari, 2015 [70] } & $\mathrm{P}$ & Preterm infants with $\mathrm{GA}<34$ weeks & Bacillus clausii & \multirow{4}{*}{ OMM, DHM } & \multirow{4}{*}{ Sterile water } & \multirow{4}{*}{$180 \mathrm{~mL} / \mathrm{kg} /$ day } \\
\hline & DB & $\begin{array}{l}\text { Excluded if: NEC, congenital anomaly, } \\
\text { outborn and }>10 \text { days of with sepsis }\end{array}$ & D: $2.4 \times 10^{9} \mathrm{CFU} /$ day & & & \\
\hline & $\mathrm{R}$ & $\begin{array}{l}\text { Stratified as extreme preterm } \\
(\text { GA } 27-30+6) \text { and very preterm } \\
(\text { GA } 31-33+6)\end{array}$ & $\begin{array}{l}\text { S: by day } 5 \text { in asymptomatic and by day } 10 \text { in } \\
\text { symptomatic infants }\end{array}$ & & & \\
\hline & $\mathrm{C}$ & & $\begin{array}{l}\text { E: } 6 \text { weeks of age, discharge or death (whichever } \\
\text { occurred first) }\end{array}$ & & & \\
\hline \multirow{5}{*}{ Totsu, 2014 [21] } & $\mathrm{P}$ & \multirow{5}{*}{ Infants with $\mathrm{BW}<1500 \mathrm{~g}$} & B. bifidum & \multirow{5}{*}{ HM, FM } & \multirow{5}{*}{ Dextrin } & \multirow{5}{*}{$\begin{array}{l}\text { Postnatal day at } \\
\text { which the amount of } \\
\text { enteral feeding } \\
\text { exceeded } \\
100 \mathrm{~mL} / \mathrm{kg} / \text { day }\end{array}$} \\
\hline & DB & & D: $2.5 \times 10^{9} \mathrm{CFU}$, divided in two doses & & & \\
\hline & CLR & & S: within $48 \mathrm{~h}$ after birth & & & \\
\hline & C & & E: body weight $2000 \mathrm{~g}$ & & & \\
\hline & Multic. & & & & & \\
\hline \multirow{4}{*}{$\begin{array}{l}\text { Van Niekerk, } \\
2014[22]\end{array}$} & $\mathrm{P}$ & \multirow{4}{*}{$\begin{array}{c}\text { Preterm infants with } \mathrm{GA}<34 \text { weeks and } \\
\text { BW }<1250 \mathrm{~g} \text {, exposed and non-exposed } \\
\text { to HIV (only infants unexposed to HIV } \\
\text { are included in the meta-analysis) }\end{array}$} & L. rhamnosus, B. infantis & \multirow{4}{*}{$\mathrm{HM}$} & \multirow{4}{*}{ MCT oil } & \multirow{4}{*}{$\begin{array}{l}\text { "when infants no } \\
\text { longer required the } \\
\text { use of IV fluids" }\end{array}$} \\
\hline & DB & & D: $0.35 \times 10^{9}$ CFU each probiotic & & & \\
\hline & $\mathrm{R}$ & & S: start of enteral feeding & & & \\
\hline & C & & E: day 28 postconceptual age & & & \\
\hline
\end{tabular}


Data from 359 infants in the probiotic group and 360 infants in the control group were evaluated: probiotic use was associated with a reduction in the time for FEF achievement (MD - 3.15 days (95\% CI $-5.25 /-1.05), p=0.003$; Figure 2a). The funnel plot did not show any clear asymmetry (Figure $2 b$ ).

$2 a$

\begin{tabular}{|c|c|c|c|c|c|c|c|c|c|c|c|c|}
\hline \multirow[b]{2}{*}{ Study or Subgroup } & \multicolumn{3}{|c|}{ Probiotic } & \multicolumn{3}{|c|}{ Control } & \multicolumn{3}{|c|}{ Mean Difference } & \multirow{2}{*}{\multicolumn{2}{|c|}{$\begin{array}{c}\text { Mean Difference } \\
\text { IV, Random, } 95 \% \mathrm{Cl}\end{array}$}} & \\
\hline & Mean & SD & Total & Mean & SD & Total & Weight & IV, Random, $95 \% \mathrm{Cl}$ & & & & \\
\hline Braga, 2011 & 15.2 & 5.2 & 119 & 17.4 & 5.7 & 112 & $21.9 \%$ & $-2.20[-3.61,-0.79]$ & & $\longrightarrow$ & & \\
\hline Manzoni, 2006 & 15 & 8 & 39 & 17 & 9 & 41 & $13.8 \%$ & $-2.00[-5.73,1.73]$ & & & & \\
\hline Roy, 2014 & 11.22 & 5.04 & 56 & 15.41 & 8.07 & 56 & $18.1 \%$ & $-4.19[-6.68,-1.70]$ & & & & \\
\hline Samanta, 2009 & 13.76 & 2.28 & 91 & 19.2 & 2.02 & 95 & $23.8 \%$ & $-5.44[-6.06,-4.82]$ & & $\rightarrow$ & & \\
\hline Van Niekerk, 2014 & 9.63 & 2.42 & 54 & 11.14 & 4.15 & 56 & $22.4 \%$ & $-1.51[-2.77,-0.25]$ & & $\rightarrow-$ & & \\
\hline Total $(95 \% \mathrm{Cl})$ & & & 359 & & & 360 & $100.0 \%$ & $-3.15[-5.25,-1.05]$ & & & & \\
\hline $\begin{array}{l}\text { Heterogeneity. Tau } \\
\text { Test for overall effec }\end{array}$ & $\begin{array}{l}4.71 ; C \\
Z=2.94\end{array}$ & $\begin{array}{l}\mathrm{i}^{2}=4 \\
(\mathrm{P}=\mathrm{C}\end{array}$ & $\begin{array}{l}1.69, \mathrm{df} \\
.003)\end{array}$ & $f=4(F$ & $\alpha 0.0$ & $11) ; 1^{2}$ & $=90 \%$ & & -10 & $\begin{array}{l}-5 \\
\text { avours probiotics }\end{array}$ & Favours co & $\begin{array}{l}1 \\
5 \\
\text { ontrols }\end{array}$ \\
\hline
\end{tabular}

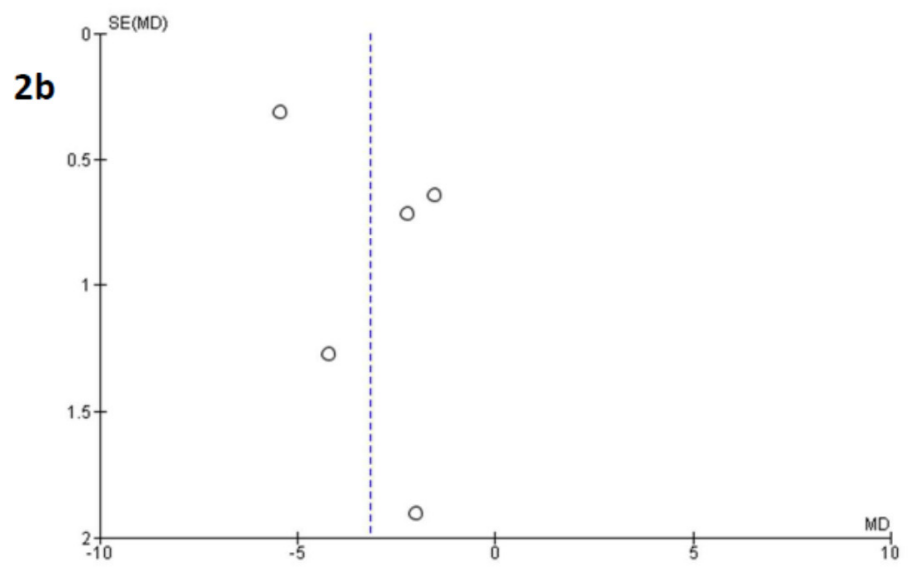

Figure 2. Forest plot (2a) and funnel plot (2b) showing the association between the use of probiotics and achievement of full enteral feeding in exclusively human milk-fed preterm infants. IV: inverse variance method.

Three studies were not included in the meta-analysis because data on FEF were not available as mean \pm SD $[41,61,70]$. One study reported the use of Bacillus clausii in preterm infants with GA $<34$ weeks, fed expressed breast milk or DHM [70] and stratified as extreme preterm (GA 27-30 + 6 weeks) and very preterm (GA 31-33 + 6 weeks). In both groups, probiotic use was associated with a reduced time to achieve FEF (risk ratio 0.82 (95\% CI 0.74-0.88) and 0.67 (95\% CI 0.32-0.77), respectively).

The other two studies reported probiotic use in exclusively formula-fed infants: in the study by Costalos et al., infants born at 28-32 weeks gestation and fed exclusively preterm formula received Saccharomyces boulardii or placebo for approximately 30 days [41]. In the study by Stratiki et al., formula-fed infants with a similar gestational age (27-32 weeks) received Bifidobacterium lactis vs. no treatment [61]. Neither of these two studies reported any significant difference between groups in terms of time to FEF achievement.

All the studies included in the meta-analysis, except one [50], recruited exclusively infants with birth weight $<1500 \mathrm{~g}$. The study by Roy et al. [50] reported specific data for extremely low birth weight (ELBW) infants: time to reach FEF in ELBW infants treated with probiotics was significantly lower than in controls (mean \pm SD $13.22 \pm 5.04$ vs. $17.41 \pm 8.07$, respectively, $p=0.014$ ). None of the studies included in the meta-analysis reported separate data on intrauterine growth restricted (IUGR) infants.

In all the studies, except one [56], a probiotic mix was used: the meta-analysis performed after the exclusion of the study by Manzoni et al., where a single-strain product containing Lactobacillus GG was used, confirmed the results of the overall analysis (MD -3.33 days $(95 \% \mathrm{CI}-5.63 /-1.04)$, $p \leqslant 0.004)$. 


\section{Methodological Study Quality}

Evaluation of the quality of the studies included in the meta-analysis according to the risk of bias tool as proposed by the Cochrane Collaboration [19] is shown in Table 2.

Table 2. Evaluation of the quality of the studies included in the meta-analysis according to the risk of bias tool as proposed by the Cochrane collaboration.

\begin{tabular}{ccccccc}
\hline Study & $\begin{array}{c}\text { Random Sequence } \\
\text { Generation }\end{array}$ & $\begin{array}{c}\text { Allocation } \\
\text { Concealment }\end{array}$ & Blinding & $\begin{array}{c}\text { Incomplete } \\
\text { Outcome Data }\end{array}$ & $\begin{array}{c}\text { Selective Outcome } \\
\text { Reporting }\end{array}$ & $\begin{array}{c}\text { Other Sources } \\
\text { of Bias }\end{array}$ \\
\hline Braga, 2011 [60] & Low & Low & Low & Low & Unclear & Low \\
Manzoni, 2006 [56] & Low & Low & Low & Unclear & Unclear & Low \\
Roy, 2014 [50] & Low & Unclear & Low & Low & Unclear & Unclear \\
Samanta, 2008 [38] & Low & Low & Low & Unclear & Unclear & Unclear \\
Van Niekerk, 2014 [22] & Low & Unclear & Low & Unclear & Unclear & Unclear \\
\hline
\end{tabular}

\section{Discussion}

The present meta-analysis shows that the use of probiotics in preterm, VLBW infants fed exclusively HM is associated with 3-days reduction in the time to FEF achievement. The only two studies included in the present systematic review in which infants were exclusively formula-fed did not report any difference between the probiotic and the control group.

The single previous meta-analysis investigating FEF as primary outcome showed an overall smaller reduction in the time to FEF achievement, but did not report separate data for HM-fed and formula-fed infants [6]. The studies included in the meta-analysis by Athalye-Jape et al. are almost the same as those included in our systematic review; quite surprisingly, in the majority of the studies included in these two reviews, both HM and formula-fed infants were recruited, but no detailed information on the relationship between type of feeding and outcome was provided.

Type of feeding might modulate the relationship between probiotics and neonatal clinical outcome [14]. It has been previously shown that HM feeding is associated with shorter time to achieve FEF compared to formula feeding [5]. Our meta-analysis, which included only studies where infants were exclusively HM-fed, showed a significant reduction in the time to achieve FEF attributable to probiotics. Despite the limitation given by the small number of studies, a probiotic-related 3-days reduction in time to achieve FEF in preterm infants fed exclusively $\mathrm{HM}$ has strong clinical implications and deserves further consideration. When OMM is not available or contraindicated, the use of pasteurized DHM is recommended for preterm infants: pasteurization inactivates most viral and bacterial agents, but at the same time affects some nutritional and immunological properties of HM, including endogenous probiotics [71]. It can be speculated that the beneficial effect of probiotics documented in exclusively HM-fed infants could be attributed to a synergic action exerted by the prebiotic components of HM and the exogenous probiotic, which partially restores the symbiotic properties of naïve HM [72]. In the present meta-analysis, no separate data for OMM-fed and DHM-fed infants were available; for this reason, it is not possible to clarify whether the beneficial effect of $\mathrm{HM}$ on FEF achievement applies both to OMM and to DHM.

Heterogeneity among included studies was high; however, given the small number of papers, our ability to explore sources of heterogeneity was limited. In the five included studies, different probiotic strains were used. We aimed to perform strain-specific sub-meta-analyses, in order to clarify whether there was any probiotic product showing a significant benefit in terms of reduction in the time to achieve FEF. However, such analyses were not feasible, as none of the studies used the same probiotic strain or mix. Similarly, it was not possible to explore additional sources of heterogeneity, such as the characteristics of probiotic administration (dose, duration, infant age at probiotic initiation, etc.). In addition, we were unable to test for subgroup differences between HM-fed and formula-fed infants, which might have partially explained the different results in terms of FEF achievement. 
Apparently, studies were homogeneous in terms of included populations, as almost all of them recruited only VLBW infants. However, few data on "high-risk" infants, such as ELBW and IUGR infants, could be extrapolated from the main results of the included studies.

The use of probiotics should be weighed against their potential side effects. There are some reports about the occurrence of sepsis in preterm newborns, potentially linked to probiotic administration [73].

However, none of the studies included in the systematic review reported any side effect related to the use of probiotics.

\section{Conclusions}

According to the results of the present meta-analysis, the use of probiotics is linked to 3-days reduction in time to achieve FEF in preterm VLBW infants fed exclusively HM. If confirmed in further studies, this reduction might have strong clinical implications for this high-risk population.

Acknowledgments: No funding was received to produce the present paper.

Author Contributions: Each author listed on the manuscript has seen and approved the submission of this version of the manuscript and takes full responsibility for the manuscript. Specifically, all the authors, as part of the Task Force on Probiotics of the Italian Society of Neonatology, conceived and designed the study protocol. A.A. and L.C. performed the literature search and assessed study details, which were checked by D.G. A.A. and D.G. evaluated study quality and performed the meta-analyses. A.A. and L.C. wrote the first draft of the paper, which was critically revised by all the other authors.

Conflicts of Interest: The authors declare no conflict of interest.

\section{References}

1. Faldella, G.; Aceti, A.; Corvaglia, L. Formula milk and neurodevelopmental and cognitive outcomes: Where are we now? Early Hum. Dev. 2011, 87S, S5-S8. [CrossRef] [PubMed]

2. Hsiao, C.-C.; Tsai, M.-L.; Chen, C.-C.; Lin, H.-C. Early optimal nutrition improves neurodevelopmental outcomes for very preterm infants. Nutr. Rev. 2014, 72, 532-540. [CrossRef] [PubMed]

3. Berrington, J.E.; Stewart, C.J.; Embleton, N.D.; Cummings, S.P. Gut microbiota in preterm infants: Assessment and relevance to health and disease. Arch. Dis. Child. Fetal Neonatal Ed. 2013, 98, F286-F290. [CrossRef] [PubMed]

4. The SIFT Investigators Group. Early enteral feeding strategies for very preterm infants: Current evidence from Cochrane reviews. Arch. Dis. Child. Fetal Neonatal Ed. 2013, 98, F470-F472.

5. Corvaglia, L.; Fantini, M.P.; Aceti, A.; Gibertoni, D.; Rucci, P.; Baronciani, D.; Faldella, G. Predictors of full enteral feeding achievement in very low birth weight infants. PLoS ONE 2014, 9. [CrossRef] [PubMed]

6. Athalye-Jape, G.; Deshpande, G.; Rao, S.; Patole, S. Benefits of probiotics on enteral nutrition in preterm neonates: a systematic review. Am. J. Clin. Nutr. 2014, 100, 1508-1519. [CrossRef] [PubMed]

7. Aceti, A.; Gori, D.; Barone, G.; Callegari, M.L.; Di Mauro, A.; Fantini, M.P.; Indrio, F.; Maggio, L.; Meneghin, F.; Morelli, L.; et al. Probiotics for prevention of necrotizing enterocolitis in preterm infants: Systematic review and meta-analysis. Ital. J. Pediatr. 2015, 41, 89. [CrossRef] [PubMed]

8. Alfaleh, K.; Anabrees, J.; Bassler, D. Probiotics for prevention of necrotizing enterocolitis in preterm infants. Cochrane Database Syst. Rev. 2014, CD005496. [CrossRef] [PubMed]

9. Rao, S.C.; Athalye-jape, G.K.; Deshpande, G.C.; Simmer, K.N.; Patole, S.K. Probiotic supplementation and late-onset sepsis in preterm infants: A meta-analysis. Pediatrics 2016, 137, e20153684. [CrossRef] [PubMed]

10. Sanders, M.E.; Guarner, F.; Guerrant, R.; Holt, P.R.; Quigley, E.M.M.; Sartor, R.B.; Sherman, P.M.; Mayer, E.A. An update on the use and investigation of probiotics in health and disease. Gut 2013, 62, 787-796. [CrossRef] [PubMed]

11. Murguía-Peniche, T.; Mihatsch, W.A.; Zegarra, J.; Supapannachart, S.; Ding, Z.-Y.; Neu, J. Intestinal mucosal defense system, Part 2. Probiotics and prebiotics. J. Pediatr. 2013, 162, S64-S71. [CrossRef] [PubMed]

12. Martin, C.R.; Walker, W.A. Probiotics: Role in pathophysiology and prevention in necrotizing enterocolitis. Semin. Perinatol. 2008, 32, 127-137. [CrossRef] [PubMed]

13. Carlisle, E.M.; Morowitz, M.J. The intestinal microbiome and necrotizing enterocolitis. Curr. Opin. Pediatr. 2013, 25, 382-387. [CrossRef] [PubMed] 
14. Repa, A.; Thanhaeuser, M.; Endress, D.; Weber, M.; Kreissl, A.; Binder, C.; Berger, A.; Haiden, N. Probiotics (Lactobacillus acidophilus and Bifidobacterium bifidum) prevent NEC in VLBW infants fed breast milk but not formula. Pediatr. Res. 2015, 77, 381-388. [CrossRef] [PubMed]

15. Moher, D.; Liberati, A.; Tetzlaff, J.; Altman, D.G. Preferred reporting items for systematic reviews and meta-analyses: The PRISMA statement. PLoS Med. 2009, 6, e1000097. [CrossRef] [PubMed]

16. PubMed. Available online: http://www.ncbi.nlm.nih.gov/pubmed (accessed on 28 July 2016).

17. Cochrane Library. Available online: http://www.cochranelibrary.com/ (accessed on 28 July 2016).

18. Embase. Available online: http://store.elsevier.com/en_US/info/30800006 (accessed on 28 July 2016).

19. Higgins, J.P.; Green, S. Cochrane Handbook for Systematic Reviews of Interventions; The Cochrane Collaboration: London, UK, 2011.

20. RevMan Software. Available online: http://tech.cochrane.org/revman/download (accessed on 28 July 2016).

21. Totsu, S.; Yamasaki, C.; Terahara, M.; Uchiyama, A.; Kusuda, S. Bifidobacterium and enteral feeding in preterm infants: Cluster-randomized trial. Pediatr. Int. 2014, 56, 714-719. [CrossRef] [PubMed]

22. Van Niekerk, E.; Kirsten, G.F.; Nel, D.G.; Blaauw, R. Probiotics, feeding tolerance, and growth: A comparison between HIV-exposed and unexposed very low birth weight infants. Nutrition 2014, 30, 645-653. [CrossRef] [PubMed]

23. Patole, S.; Keil, A.D.; Chang, A.; Nathan, E.; Doherty, D.; Simmer, K.; Esvaran, M.; Conway, P. Effect of Bifidobacterium breve M-16V supplementation on fecal bifidobacteria in preterm neonates-A randomised double blind placebo controlled trial. PLoS ONE 2014, 9, e89511. [CrossRef] [PubMed]

24. Oncel, M.Y.; Sari, F.N.; Arayici, S.; Guzoglu, N.; Erdeve, O.; Uras, N.; Oguz, S.S.; Dilmen, U. Lactobacillus Reuteri for the prevention of necrotising enterocolitis in very low birthweight infants: A randomised controlled trial. Arch. Dis. Child. Fetal Neonatal Ed. 2014, 99, F110-F115. [CrossRef] [PubMed]

25. Jacobs, S.E.; Tobin, J.M.; Opie, G.F.; Donath, S.; Tabrizi, S.N.; Pirotta, M.; Morley, C.J.; Garland, S.M. Probiotic effects on late-onset sepsis in very preterm infants: A randomized controlled trial. Pediatrics 2013, 132, 1055-1062. [CrossRef] [PubMed]

26. Serce, O.; Benzer, D.; Gursoy, T.; Karatekin, G.; Ovali, F. Efficacy of saccharomyces boulardii on necrotizing enterocolitis or sepsis in very low birth weight infants: A randomised controlled trial. Early Hum. Dev. 2013, 89, 1033-1036. [CrossRef] [PubMed]

27. Demirel, G.; Erdeve, O.; Celik, I.H.; Dilmen, U. Saccharomyces boulardii for prevention of necrotizing enterocolitis in preterm infants: A randomized, controlled study. Acta Paediatr. 2013, 102, 560-565. [CrossRef] [PubMed]

28. Rojas, M.A.; Lozano, J.M.; Rojas, M.X.; Rodriguez, V.A.; Rondon, M.A.; Bastidas, J.A.; Perez, L.A.; Rojas, C.; Ovalle, O.; Garcia-Harker, J.E.; et al. Prophylactic probiotics to prevent death and nosocomial infection in preterm infants. Pediatrics 2012, 130, e1113-e1120. [CrossRef] [PubMed]

29. Sari, F.N.; Eras, Z.; Dizdar, E.A.; Erdeve, O.; Oguz, S.S.; Uras, N.; Dilmen, U. Do oral probiotics affect growth and neurodevelopmental outcomes in very low-birth-weight preterm infants? Am. J. Perinatol. 2012, 29, 579-586. [CrossRef] [PubMed]

30. Fernández-Carrocera, L.A.; Solis-Herrera, A.; Cabanillas-Ayón, M.; Gallardo-Sarmiento, R.B.; García-Pérez, C.S.; Montaño-Rodríguez, R.; Echániz-Aviles, M.O.L. Double-blind, randomised clinical assay to evaluate the efficacy of probiotics in preterm newborns weighing less than $1500 \mathrm{~g}$ in the prevention of necrotising enterocolitis. Arch. Dis. Child. Fetal Neonatal Ed. 2013, 98, F5-F9. [CrossRef] [PubMed]

31. Havranek, T.; Al-Hosni, M.; Armbrecht, E. Probiotics supplementation increases intestinal blood flow velocity in extremely low birth weight preterm infants. J. Perinatol. 2013, 33, 40-44. [CrossRef] [PubMed]

32. Chrzanowska-Liszewska, D.; Seliga-Siwecka, J.; Kornacka, M.K. The effect of Lactobacillus rhamnosus GG supplemented enteral feeding on the microbiotic flora of preterm infants-double blinded randomized control trial. Early Hum. Dev. 2012, 88, 57-60. [CrossRef] [PubMed]

33. Campeotto, F.; Suau, A.; Kapel, N.; Magne, F.; Viallon, V.; Ferraris, L.; Waligora-Dupriet, A.-J.; Soulaines, P.; Leroux, B.; Kalach, N.; Dupont, C.; Butel, M.-J. A fermented formula in pre-term infants: Clinical tolerance, gut microbiota, down-regulation of faecal calprotectin and up-regulation of faecal secretory IgA. Br. J. Nutr. 2011, 105, 1843-1851. [CrossRef] [PubMed]

34. Sari, F.N.; Dizdar, E.A.; Oguz, S.; Erdeve, O.; Uras, N.; Dilmen, U. Oral probiotics: Lactobacillus sporogenes for prevention of necrotizing enterocolitis in very low-birth weight infants: A randomized, controlled trial. Eur. J. Clin. Nutr. 2011, 65, 434-439. [CrossRef] [PubMed] 
35. Indrio, F.; Riezzo, G.; Raimondi, F.; Bisceglia, M.; Cavallo, L.; Francavilla, R. Effects of probiotic and prebiotic on gastrointestinal motility in newborns. J. Physiol. Pharmacol. 2009, 60, 27-31. [PubMed]

36. Mihatsch, W.A.; Vossbeck, S.; Eikmanns, B.; Hoegel, J.; Pohlandt, F. Effect of Bifidobacterium lactis on the incidence of nosocomial infections in very-low-birth-weight infants: A randomized controlled trial. Neonatology 2010, 98, 156-163. [CrossRef] [PubMed]

37. Rougé, C.; Piloquet, H.; Butel, M.-J.; Berger, B.; Rochat, F.; Ferraris, L.; Des Robert, C.; Legrand, A.; de la Cochetiere, M.-F.; N'Guyen, J.-M.; et al. Oral supplementation with probiotics in very-low-birth-weight preterm infants: A randomized, double-blind, placebo-controlled trial. Am. J. Clin. Nutr. 2009, 89, 1828-1835. [CrossRef] [PubMed]

38. Samanta, M.; Sarkar, M.; Ghosh, P.; Ghosh, J.K.; Sinha, M.K.; Chatterjee, S. Prophylactic probiotics for prevention of necrotizing enetrocolitis in very low birth weight newborns. J. Trop. Pediatr. 2008, 55, 128-131. [CrossRef] [PubMed]

39. Lin, H.-C.; Hsu, C.-H.; Chen, H.-L.; Chung, M.-Y.; Hsu, J.-F.; Lien, R.; Tsao, L.-Y.; Chen, C.-H.; Su, B.-H. Oral probiotics prevent necrotizing enterocolitis in very low birth weight preterm infants: A multicenter, randomized, controlled trial. Pediatrics 2008, 122, 693-700. [CrossRef] [PubMed]

40. Bin-Nun, A.; Bromiker, R.; Wilschanski, M.; Kaplan, M.; Rudensky, B.; Caplan, M.; Hammerman, C. Oral probiotics prevent necrotizing enterocolitis in very low birth weight neonates. J. Pediatr. 2005, 147, $192-196$. [CrossRef] [PubMed]

41. Costalos, C.; Skouteri, V.; Gounaris, A.; Sevastiadou, S.; Triandafilidou, A.; Ekonomidou, C.; Kontaxaki, F.; Petrochilou, V. Enteral feeding of premature infants with Saccharomyces boulardii. Early Hum. Dev. 2003, 74, 89-96. [CrossRef]

42. Dani, C.; Biadaioli, R.; Bertini, G.; Martelli, E.; Rubaltelli, F.F. Probiotics feeding in prevention of urinary tract infection, bacterial sepsis and necrotizing enterocolitis in preterm infants. Biol. Neonate 2002, 82, 103-108. [CrossRef] [PubMed]

43. Stansbridge, E.M.; Walker, V.; Hall, M.A.; Smith, S.L.; Millar, M.R.; Bacon, C.; Chen, S. Effects of feeding premature infants with Lactobacillus GG on gut fermentation. Arch. Dis. Child. 1993, 69, 488-492. [CrossRef] [PubMed]

44. Millar, M.R.; Bacon, C.; Smith, S.L.; Walker, V.; Hall, M.A. Enteral feeding of premature infants with Lactobacillus GG. Arch. Dis. Child. 1993, 69, 483-487. [CrossRef] [PubMed]

45. Indrio, F.; Riezzo, G.; Raimondi, F.; Bisceglia, M.; Cavallo, L.; Francavilla, R. The effects of probiotics on feeding tolerance, bowel habits, and gastrointestinal motility in preterm newborns. J. Pediatr. 2008, 152, 801-806. [CrossRef] [PubMed]

46. Al-Hosni, M.; Duenas, M.; Hawk, M.; Stewart, L.A.; Borghese, R.A.; Cahoon, M.; Atwood, L.; Howard, D.; Ferrelli, K.; Soll, R. Probiotics-supplemented feeding in extremely low-birth-weight infants. J. Perinatol. 2012, 32, 253-259. [CrossRef] [PubMed]

47. Savino, F.; Ceratto, S.; Poggi, E.; Cartosio, M.E.; Cordero di Montezemolo, L.; Giannattasio, A. Preventive effects of oral probiotic on infantile colic: A prospective, randomised, blinded, controlled trial using Lactobacillus reuteri DSM 17938. Benef. Microbes 2014. [CrossRef]

48. Saengtawesin, V.; Tangpolkaiwalsak, R.; Kanjanapattankul, W. Effect of oral probiotics supplementation in the prevention of necrotizing enterocolitis among very low birth weight preterm infants. J. Med. Assoc. Thail. 2014, 97, S20-S25.

49. Dilli, D.; Aydin, B.; Fettah, N.; Özyazıcı, E.; Beken, S.; Zenciroğlu, A.; Okumuş, N.; Özyurt, B.; İpek, M.; Akdağ, A.; et al. The propre-save study: Effects of probiotics and prebiotics alone or combined on necrotizing enterocolitis in very low birth weight infants. J. Pediatr. 2015, 28, 1537-1541. [CrossRef] [PubMed]

50. Roy, A.; Chaudhuri, J.; Sarkar, D.; Ghosh, P.; Chakraborty, S. Role of enteric supplementation of Probiotics on late-onset sepsis by Candida species in preterm low birth weight neonates: A randomized, double blind, placebo-controlled trial. N. Am. J. Med. Sci. 2014, 6, 50-57. [PubMed]

51. Oncel, M.Y.; Arayici, S.; Sari, F.N.; Simsek, G.K.; Yurttutan, S.; Erdeve, O.; Saygan, S.; Uras, N.; Oguz, S.S.; Dilmen, U. Comparison of Lactobacillus reuteri and nystatin prophylaxis on Candida colonization and infection in very low birth weight infants. J. Matern. Neonatal Med. 2014, 1-5. [CrossRef]

52. Millar, M.; Wilks, M.; Fleming, P.; Costeloe, K. Should the use of probiotics in the preterm be routine? Arch. Dis. Child. Fetal Neonatal Ed. 2012, 97, F70-F74. [CrossRef] [PubMed] 
53. Rinaldi, M.; Manzoni, P.; Meyer, M.; Casa, E.D.; Pugni, L.; Mosca, F.; Stolfi, I.; Messner, H.; Memo, L.; Laforgia, N.; et al. Bovine lactoferrin supplementation for prevention of necrotising enterocolitis in preterm very-low-birth-weight neonates: A randomised trial. Early Hum. Dev. 2012, 88, S102. [CrossRef]

54. Manzoni, P.; Meyer, M.; Stolfi, I.; Rinaldi, M.; Cattani, S.; Pugni, L.; Romeo, M.G.; Messner, H.; Decembrino, L.; Laforgia, N.; et al. Bovine lactoferrin supplementation for prevention of necrotizing enterocolitis in very-low-birth-weight neonates: A randomized clinical trial. Early Hum. Dev. 2014, 90, S60-S65. [CrossRef]

55. Benor, S.; Marom, R.; Tov, A.B.; Domany, K.A.; Zaidenberg-Israeli, G.; Dollberg, S. Probiotic supplementation in mothers of very low birth weight infants. Am. J. Perinatol. 2014, 31, 497-504. [PubMed]

56. Manzoni, P.; Mostert, M.; Leonessa, M.L.; Priolo, C.; Farina, D.; Monetti, C.; Latino, M.A.; Gomirato, G. Oral supplementation with Lactobacillus casei subspecies rhamnosus prevents enteric colonization by Candida species in preterm neonates: A randomized study. Clin. Infect. Dis. 2006, 42, 1735-1742. [CrossRef] [PubMed]

57. Kitajima, H.; Sumida, Y.; Tanaka, R.; Yuki, N.; Takayama, H.; Fujimura, M. Early administration of Bifidobacterium breve to preterm infants: Randomised controlled trial. Arch. Dis. Child. Fetal Neonatal Ed. 1997, 76, F101-F107. [CrossRef] [PubMed]

58. Mohan, R.; Koebnick, C.; Schildt, J.; Schmidt, S.; Mueller, M.; Possner, M.; Radke, M.; Blaut, M. Effects of Bifidobacterium lactis Bb12 supplementation on intestinal microbiota of preterm infants: A double-blind, placebo-controlled, randomized study. J. Clin. Microbiol. 2006, 44, 4025-4031. [CrossRef] [PubMed]

59. Lin, H.-C.; Su, B.-H.; Chen, A.-C.; Lin, T.-W.; Tsai, C.-H.; Yeh, T.-F.; Oh, W. Oral probiotics reduce the incidence and severity of necrotizing enterocolitis in very low birth weight infants. Pediatrics 2005, 115, 1-4. [PubMed]

60. Braga, T.D.; da Silva, G.A.P.; de Lira, P.I.; de Carvalho Lima, M. Efficacy of Bifidobacterium breve and Lactobacillus casei oral supplementation on necrotizing enterocolitis in very-low-birth-weight preterm infants: A double-blind, randomized, controlled trial. Am. J. Clin. Nutr. 2011, 93, 81-86. [CrossRef] [PubMed]

61. Stratiki, Z.; Costalos, C.; Sevastiadou, S.; Kastanidou, O.; Skouroliakou, M.; Giakoumatou, A.; Petrohilou, V. The effect of a bifidobacter supplemented bovine milk on intestinal permeability of preterm infants. Early Hum. Dev. 2007, 83, 575-579. [CrossRef] [PubMed]

62. Manzoni, P.; Rinaldi, M.; Cattani, S.; Pugni, L.; Romeo, M.G.; Messner, H. Bovine lactoferrin supplementation for prevention of late-onset sepsis in very low-birth-weight neonates. JAMA 2009, 302, 1421-1428. [CrossRef] [PubMed]

63. Li, Y.; Shimizu, T.; Hosaka, A.; Kaneko, N.; Ohtsuka, Y.; Yamashiro, Y. Effects of bifidobacterium breve supplementation on intestinal flora of low birth weight infants. Pediatr. Int. 2004, 46, 509-515. [CrossRef] [PubMed]

64. Costeloe, K.; Hardy, P.; Juszczak, E.; Wilks, M.; Millar, M.R. Bifidobacterium breve BBG-001 in very preterm infants: A randomised controlled phase 3 trial. Lancet 2015, 387, 649-660. [CrossRef]

65. Dutta, S.; Ray, P.; Narang, A. Comparison of stool colonization in premature infants by three dose regimes of a probiotic combination: A randomized controlled trial. Am. J. Perinatol. 2015, 32, 733-740. [PubMed]

66. Hays, S.; Jacquot, A.; Gauthier, H.; Kempf, C.; Beissel, A.; Pidoux, O.; Jumas-Bilak, E.; Decullier, E.; Lachambre, E.; Beck, L.; et al. Probiotics and growth in preterm infants: A randomized controlled trial, PREMAPRO study. Clin. Nutr. 2014. [CrossRef] [PubMed]

67. Romeo, M.G.; Romeo, D.M.; Trovato, L.; Oliveri, S.; Palermo, F.; Cota, F.; Betta, P. Role of probiotics in the prevention of the enteric colonization by Candida in preterm newborns: Incidence of late-onset sepsis and neurological outcome. J. Perinatol. 2011, 31, 63-69. [CrossRef] [PubMed]

68. Hikaru, U.; Koichi, S.; Yayoi, S.; Hiromici, S.; Hiroaki, S.; Yoshkazu, O.; Seigo, A.; Satoru, N.; Toshiaki, S.; Yuichiro, Y. Bifidobacteria prevents preterm infants from developing infection and sepsis. Int. J. Probiotics Prebiotics 2010, 5, 33-36.

69. Patole, S.K.; Keil, A.D.; Nathan, E.; Doherty, D.; Esvaran, M.; Simmer, K.N.; Conway, P. Effect of Bifidobacterium breve $\mathrm{M}-16 \mathrm{~V}$ supplementation on fecal bifidobacteria in growth restricted very preterm infants -analysis from a randomised trial. J. Matern. Fetal Neonatal Med. 2016. [CrossRef] [PubMed]

70. Tewari, V.V.; Dubey, S.K.; Gupta, G. Bacillus clausii for prevention of late-onset sepsis in preterm infants: A randomized controlled trial. J. Trop. Pediatr. 2015, 61, 377-385. [CrossRef] [PubMed] 
71. Bertino, E.; Giuliani, F.; Baricco, M.; Di Nicola, P.; Peila, C.; Vassia, C.; Chiale, F.; Pirra, A.; Cresi, F.; Martano, C.; Coscia, A. Benefits of donor milk in the feeding of preterm infants. Early Hum. Dev. 2013, 89, S3-S6. [CrossRef] [PubMed]

72. Zivkovic, A.M.; German, J.B.; Lebrilla, C.B.; Mills, D.A. Human milk glycobiome and its impact on the infant gastrointestinal microbiota. Proc. Natl. Acad. Sci. USA 2011, 108, 4653-4658. [CrossRef] [PubMed]

73. Bertelli, C.; Pillonel, T.; Torregrossa, A.; Prod'hom, G.; Fischer, C.J.; Greub, G.; Giannoni, E. Bifidobacterium longum bacteremia in preterm infants receiving probiotics. Clin. Infect. Dis. 2015, 60, 924-927. [CrossRef] [PubMed]

(C) 2016 by the authors; licensee MDPI, Basel, Switzerland. This article is an open access article distributed under the terms and conditions of the Creative Commons Attribution (CC-BY) license (http://creativecommons.org/licenses/by/4.0/). 